\title{
$\mathrm{MoO}_{2}$ 储锂性能及循环容量反常特性的第一性原理研究
}

\author{
吉晓刘雅雯余晓伟杨凯吴江滨缪灵* 江建军
}

(华中科技大学 光学与电子信息学院 武汉 430074)

\begin{abstract}
摘要 基于第一性原理, 对 $\mathrm{MoO}_{2}$ 作为电极材料的储锂性能进行了计算, 并探讨了其储锂容量在一定循环次数内呈上 升的反常现象微观机理. 计算了 $\mathrm{MoO}_{2}$ 材料中 $\mathrm{Li}$ 的单键能, 态密度(DOS)及其嵌锂电压, 结果表明 $\mathrm{MoO}_{2}$ 中 $\mathrm{Li}$ 的吸附 能较大, 储锂结构稳定. 嵌锂结构呈金属性, 嵌锂电压变化规律与文献实验结果一致. 针对循环容量反常特性, 计算了 $\mathrm{Mo}$ 的空位形成能, $\mathrm{LiMoO}_{2}$ 的差分电荷密度以及电荷布居情况, 计算结果表明 $\mathrm{Li}$ 的嵌入能为 $\mathrm{O}$ 提供电荷, 减弱了 $\mathrm{Mo}-\mathrm{O}$ 键间的相互作用, 另一方面嵌入的 $\mathrm{Li}$ 能减弱 Mo 空位形成后的电荷极化作用, 从而大大降低 Mo 空位的形成能. 形成的 Mo 空位能为 $\mathrm{Li}$ 的嵌入提供了新的吸附位点, 提高了嵌锂的容量. 计算结果与实验符合得很好, 能为电极材料 储锂性能的改善提供一定的理论指导.
\end{abstract}

关键词 第一性原理; 锂电池; $\mathrm{MoO}_{2}$; 循环容量

\section{First Principles Study on the Li Storage Performance of $\mathrm{MoO}_{2}$}
Ji, Xiao
Liu, Yawen
Yu, Xiaowei
Yang, Kai
Wu, Jiangbin
Miao, Ling* Jiang, Jianjun
(School of Optical and Electronic Information, HuaZhong University of Science and Technology, Wuhan 430074)

\begin{abstract}
Based on first principle calculations, $\mathrm{MoO}_{2}$ as electrode for lithium storage performance was calculated, and explored the microscopic mechanism of the anomaly phenmenon that the lithium storage capacity of $\mathrm{MoO}_{2}$ increase within a certain number of cycles. All of our calculations, including geometry and electronic structure calculation are performed by using density function theory on the basis of projector augmented wave (PAW) method implemented in the VASP package. Generalized gradient approximation (GGA) was employed to describe the exchange-correlation interactions. The kinetic energy cutoff of electron wave functions is $400 \mathrm{eV}$ in the calculation. The structure relaxations were carried out until all the atomic forces in each ion were less than $0.02 \mathrm{eV} / \AA$. We calculated average binding energy, density of states (DOS) and average cell voltage. We find that the binding energy is large which means the structure of $\mathrm{MoO}_{2}$ with $\mathrm{Li}$ is stable. And because the system is disordered by $\mathrm{Li}$, the average binding energy decrease as the number of lithium increase. The average cell voltages show the same law with the experimental results, which show our calculation is reasonable. To explain the unusual characteristics of the circulating volume, Mo vacancy formation energy, $\mathrm{LiMoO}_{2}$ different charge density and charge distribution are calculated. The results show that the intercalation of $\mathrm{Li}$ weakened the $\mathrm{Mo}-\mathrm{O}$ bond, on the other hand weakened the role of the charge polarization of the Mo vacancy, and thus greatly reduce the formation energy of Mo vacancy. What's more, the Mo vacancy provides new adsorption for Li, improve the capacity of lithium intercalation. In a word, the intercalation of lithium made the Mo vacancy form easily, and at the same time provides new volume for the lithium intercalation. The results are in good agreement with experimental, can provide some theoretical guidance for the improvement of the electrode materials for lithium storage.

Keywords first principle calculations; lithium ion battery; $\mathrm{MoO}_{2}$; unusual circulating volume
\end{abstract}

\section{1 引言}

锂离子电池由于体积小、重量轻、功率密度高、无 记忆效应等特点已成为当前研究热点 ${ }^{[1,2]}$, 在手机、汽车 等移动设备上也有很重要的应用, 给生产、生活带来巨 大的便利. 选择合适的电池电极材料是设计锂离子电池 的关键内容, 其中电极材料的选取指标主要包括成本、 安全性、工作电压、能量密度、功率密度以及循环寿命. 相比于其他材料, 过渡金属氧化物 $\left(\mathrm{SnO}_{2}{ }^{[3]}, \mathrm{TiO}_{2}{ }^{[4]}\right.$, $\left.\mathrm{Fe}_{2} \mathrm{O}_{3}{ }^{[5]}, \mathrm{Co}_{3} \mathrm{O}_{4}{ }^{[6 \sim 8]}, \mathrm{NiO}^{[9]}, \mathrm{MnO}_{2}{ }^{[10,11]}, \mathrm{MoO}_{3}{ }^{[12]}, \mathrm{WO}_{3}{ }^{[13]}\right)$
作为锂电池负极材料不仅来源广泛、成本较低, 而且拥 有更高的理论电化学容量, 并具有良好的循环性能, 因 而受到了广泛关注. 例如 Lei 等 ${ }^{[14]}$ 将 $\mathrm{SnO}_{2}$ 纳米阵列作为 锂离子电池电极材料, 通过实验测得其容量在 50 个循 环周期后仍保持 $607 \mathrm{mAh} / \mathrm{g}$. Arrouvel 等 ${ }^{[15]}$ 利用第一性 原理计算了 $\mathrm{Li}$ 在 $\mathrm{TiO}_{2}$ 吸附位点、扩散路径以及平均嵌 锂电压等储锂相关特性, 与实验情况符合得很好. Guo 等 ${ }^{[16]}$ 制出直径约为 $5 \mathrm{~nm}$ 的超细 $\mathrm{MoO}_{2}$ 纳米棒作为锂电 池电极材料, 实验发现这种材料不仅拥有高可逆容量和 优良循环性能, 并通过第一性原理计算讨论了 $\mathrm{MoO}_{2}$ 纳

\footnotetext{
* E-mail: miaoling@hust.edu.cn

Received October 31, 2012; published January 16, 2013.
} 
米棒容量高的原因. 值得注意的是其容量随着循环次数 的增加而上升这一有趣现象.

当前关于过渡金属氧化物用于锂离子电池电极的 研究多集中于实验, 而锂嵌入过渡金属氧化物电极的机 理的理论研究则较少. 本文将通过第一性原理计算系统 地研究 $\mathrm{MoO}_{2}$ 嵌锂体系的稳定性, 嵌锂行为, 嵌锂的电 压以及电子特性. 特别地, 针对 $\mathrm{MoO}_{2}$ 的循环容量反常 现象, 本文将电荷密度分布以及空位形成能等对 $\mathrm{MoO}_{2}$ 嵌锂结构进行深入的探讨, 以期从微观上认识 $\mathrm{MoO}_{2}$ 作 为锂电池电极的机理, 并为锂电池材料的改进以及新材 料的探究提供一定的指导.

\section{2 计算方法与模型}

本文采用基于密度泛函 ${ }^{[17,18]}$ 理论的 VASP 程序

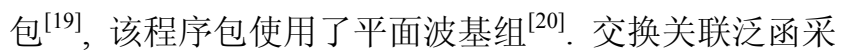
用 GGA-PBE 近似方法 ${ }^{[21,22]}$, 平面波截断能取 $400 \mathrm{eV}$. 结构优化过程中, 简约布里渊区积分 $\boldsymbol{k}$ 点选取 $3 \times 3 \times 3$ 网格. 计算中考虑了自旋极化效应, 原子受力的收玫精 度为 $0.02 \mathrm{eV} / \AA$, 总能量误差不大于 $10^{-4} \mathrm{eV}$.

本文采用单斜 $\mathrm{MoO}_{2}$ 晶胞作为研究对象, 其惯用晶 胞参数为 $a=5.6109 \AA, b=4.8562 \AA, c=5.6285 \AA, \beta=$ $120.950^{\circ[23]}$, 计算中主要采用 $2 \times 2 \times 2$ 的 $\mathrm{MoO}_{2}$ 超胞及 其不同的嵌锂结构 $\mathrm{Li}_{x} \mathrm{MoO}_{2}(x=0.25,0.5,0.75,1), \mathrm{MoO}_{2}$ 和 $\mathrm{LiMoO}_{2}$ 的计算模型示意图如图 1 所示.
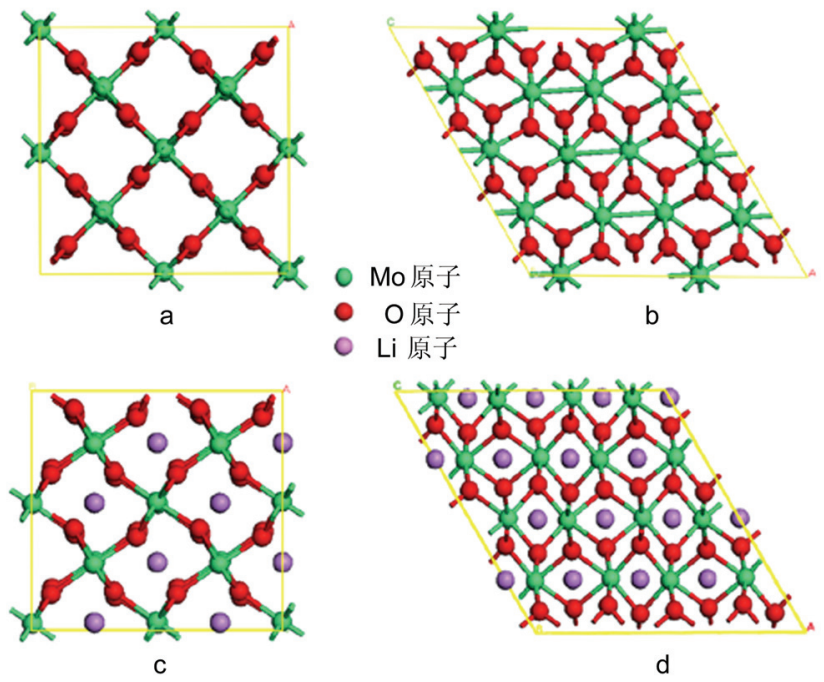

图 $1 \mathrm{MoO}_{2}$ 和 $\mathrm{LiMoO}_{2}$ 计算模型, 其中(a), (c)为[100]方向视图; (b), (d) 为 [010]方向视图

Figure 1 Atomic structures of monoclinic (a), (b) $\mathrm{MoO}_{2}$ and (c), (d) $\mathrm{LiMoO}_{2}$. (a), (c) is the [100] side view and the (b), (d) is the [010] side view

\section{3 结果与讨论}

\section{1 稳定性及平均嵌锂电压预测}

本文计算了 $\mathrm{Li}_{x} \mathrm{MoO}_{2}(x=0.25,0.5,0.75,1)$ 四种嵌 锂结构中 $\mathrm{Li}$ 的吸附能和单键能. 吸附能的计算方法为
$E_{\mathrm{b}}=\left[E\left(\mathrm{MoO}_{2}+n \mathrm{Li}\right)-n * u_{\mathrm{Li}}-E\left(\mathrm{MoO}_{2}\right)\right]$, 单键能计算公 式为 $E_{\mathrm{d}}=\left[E\left(\mathrm{MoO}_{2}+n \mathrm{Li}\right)-n * u_{\mathrm{Li}}-E\left(\mathrm{MoO}_{2}\right)\right] / n$, 其中 $E_{\mathrm{d}}$ 表示 $\mathrm{Li}$ 的单键能, $E\left(\mathrm{MoO}_{2}+n \mathrm{Li}\right)$ 表示嵌入 $n$ 个 $\mathrm{Li}$ 的 $\mathrm{MoO}_{2}$ 晶胞的总能量, $u_{\mathrm{Li}}$ 表示 $\mathrm{Li}$ 的化学势, $E\left(\mathrm{MoO}_{2}\right)$ 表示 $\mathrm{MoO}_{2}$ 晶胞的总能量. 计算得到不同嵌锂结构 $\mathrm{Li}$ 的单键 能如表 1 所示, 结果表明, 单个 $\mathrm{Li}$ 的吸附能为 $0.94 \mathrm{eV}$, 吸附能较大, 储锂结构稳定. 比较总的吸附能和单键能 可以看出, 随着 $\mathrm{Li}$ 嵌入数目的增加, 总的吸附能增加, 表明体系的稳定性增加，而单键能整体呈现下降趋势， 这是因为 $\mathrm{Li}$ 的嵌入破坏了 $\mathrm{MoO}_{2}$ 的有序性, 成键越多有 序性破坏越强烈, 单键能降低.

表 1 不同嵌锂结构的吸附能与单键能

Table 1 The binding energy and average binding energy of different structures of $\mathrm{MoO}_{2}$ with $\mathrm{Li}$

\begin{tabular}{ccccc}
\hline $\mathrm{Li}_{x} \mathrm{MoO}_{2}$ & $\mathrm{Li}_{0.25} \mathrm{MoO}_{2}$ & $\mathrm{Li}_{0.5} \mathrm{MoO}_{2}$ & $\mathrm{Li}_{0.75} \mathrm{MoO}_{2}$ & $\mathrm{LiMoO}_{2}$ \\
\hline$E_{\mathrm{b}} / \mathrm{eV}$ & 0.94 & 1.87 & 1.81 & 1.90 \\
$E_{\mathrm{d}} / \mathrm{eV}$ & 0.94 & 0.69 & 0.64 & 0.50 \\
\hline
\end{tabular}

图 2 给出了 $\mathrm{MoO}_{2}$ 和 $\mathrm{LiMoO}_{2}$ 的总态密度, 从图中 可以看出 $\mathrm{MoO}_{2}$ 和 $\mathrm{LiMoO}_{2}$ 在费米能级处 $(0 \mathrm{eV}$ 处)的态 密度均不为 0 , 均表现为金属的性质, $\mathrm{LiMoO}_{2}$ 费米能级 相对 $\mathrm{MoO}_{2}$ 费米能级右移, 且在费米能级处的态密度上 升, 说明 $\mathrm{Li}$ 的嵌入, 使得体系的费米面升高, 导电性增 强, 有利于提高电极材料的充放电速率. 平均嵌锂电压 的计算 ${ }^{[15]}$ 可以利用能斯特关系，由下式进行计算.
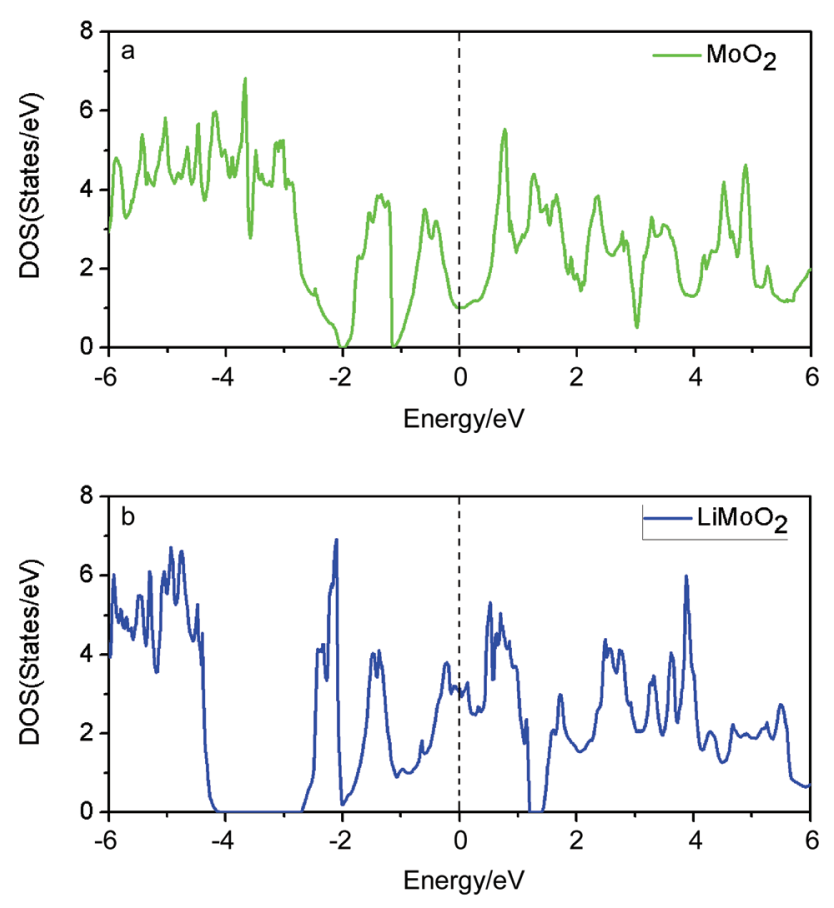

图 2 (a), (b)分别表示 $\mathrm{MoO}_{2}$ 和 $\mathrm{LiMoO}_{2}$ 的态密度(自旋向上和向下相 同, 垂直坚线表示费米能级)

Figure 2 Density of states of monoclinic (a) $\mathrm{MoO}_{2}$ and (b) $\mathrm{LiMoO}_{2}$. The Fermi level is chosen as $0 \mathrm{eV}$ 


$$
V(x)=\frac{-\Delta G}{x F}
$$

其中, $\Delta G$ 是吉布斯自由能的变化量, $F$ 是法拉第常数, $x$ 为嵌入 $\mathrm{Li}$ 的个数. 对于一般情况, 体系体积变化非常小, $P \Delta V$ 的值很小 (在 $10^{-5} \mathrm{eV}$ 左右的数量级), 故平均嵌锂 电压的公式又可以近似写成.

$$
V(x)=\frac{-\Delta E}{x F}
$$

本文通过计算, 给出了不同嵌锂结构 $\mathrm{Li}_{x} \mathrm{MoO}_{2}(x=$ $0.0625,0.125,0.25,0.5,0.75,1$ ) 的平均电压(见图 3). 从 整体来看, 随着容量的上升, 平均嵌锂电压呈逐渐下降 的趋势. 明显的可以看出, 从 $x=0.125$ 到 $x=0.5$ 部分曲 线下降很快, 而从 $x=0.5$ 到 $x=0.75$ 曲线下降较缓, 从 $x=0.75$ 往后曲线下降速度又呈现上升的趋势, 这与实 验测得的结果 ${ }^{[16]}$ 具有相同的规律, 放电平台在 $0.6 \sim 0.8$ $\mathrm{V}$, 略小于实验值 $(1.4 \sim 1.7 \mathrm{~V})$, 原因之一在于没有考虑 电子间的库伦排斥作用.

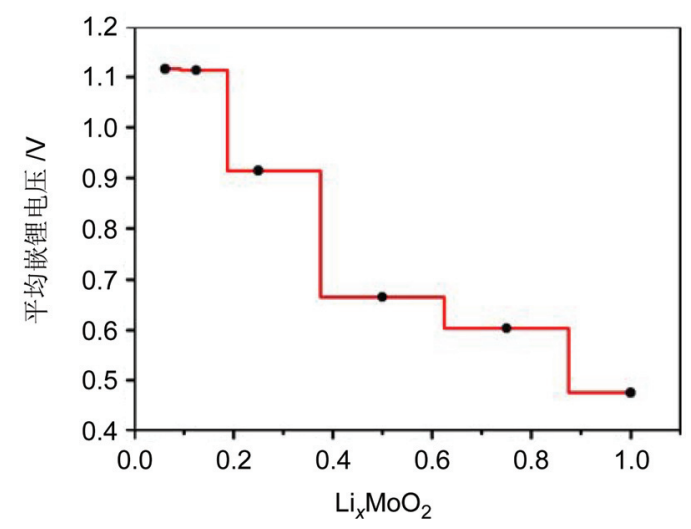

图 3 不同嵌锂结构 $\mathrm{Li}_{x} \mathrm{MoO}_{2}(x=0.0625,0.125,0.25,0.5,0.75,1)$ 的平 均嵌锂电压

Figure 3 Calculated cell voltages for different structure of $\mathrm{Li}_{x} \mathrm{MoO}_{2}$ $(x=0.0625,0.125,0.25,0.5,0.75,1)$

\section{2 反常特性微观机理探讨}

为了加深 $\mathrm{MoO}_{2}$ 在嵌锂的过程中随着循环次数增加 容量反而升高的反常情况的微观机理的理解, 本文从差 分电荷密度图和电荷布居的情况出发进行分析. 图 4 为 特定平面的差分电荷密度分布图, 可以看出, $\mathrm{Li}$ 和 $\mathrm{O}$ 之 间的电荷增加, 说明 $\mathrm{Li}$ 和 $\mathrm{O}$ 之间形成了相互作用较强 的共价键, 也从另一个侧面解释了 $\mathrm{MoO}_{2}$ 嵌锂结构稳定 的原因.

为了更直观地反映 $\mathrm{Li}, \mathrm{Mo}, \mathrm{O}$ 之间的相互作用, 本 文对 $\mathrm{MoO}_{2}$ 及 $\mathrm{LiMoO}_{2}$ 进行了 Mulliken 电荷布居的分析. 数值结果如表 2 所示, 对比 $\mathrm{MoO}_{2}$ 和 $\mathrm{LiMoO}_{2}$ 的电荷转 移情况可以看出 $\mathrm{Mo}$ 为 $\mathrm{O}$ 提供的平均电荷由 0.744 减小 为 0.684 , 即 $\mathrm{Mo}$ 和 $\mathrm{O}$ 之间的相互作用减弱. 而减弱的原 因正是由于 $\mathrm{Li}$ 为 $\mathrm{O}$ 提供了一定数量的电子. 总之, $\mathrm{Li}$ 的 嵌入能为 $\mathrm{O}$ 提供一定量的电荷, 使 $\mathrm{Mo}-\mathrm{O}$ 键作用减弱.

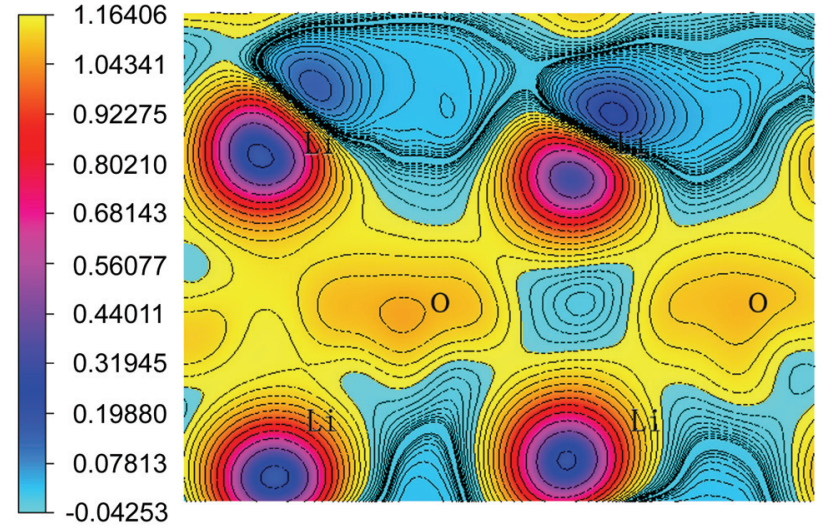

图 $4 \mathrm{LiMoO}_{2}$ 特定截面的差分电荷密度分布图

Figure 4 Different charge density of $\mathrm{LiMoO}_{2}$ in a specific plane

表 $2 \mathrm{MoO}_{2}$ 和 $\mathrm{LiMoO}_{2}$ Mulliken 电荷布居

Table 2 Mulliken charge population of $\mathrm{MoO}_{2}$ and $\mathrm{LiMoO}_{2}$

\begin{tabular}{cccc}
\hline & Mo & $\mathrm{O}$ & $\mathrm{Li}$ \\
\hline $\mathrm{MoO}_{2}$ & 0.744 & -0.372 & - \\
$\mathrm{LiMoO}_{2}$ & 0.684 & -0.502 & 0.320 \\
\hline
\end{tabular}

基于以上讨论结果, 本文分别计算了有无 $\mathrm{Li}$ 情况 下 $\mathrm{Mo}$ 和 $\mathrm{O}$ 的空位形成能 ${ }^{[1]}$, 其计算公式为 $E_{\mathrm{f}}=E(\mathrm{~V})+$ $m * u-E\left(\mathrm{MoO}_{2}+m * \mathrm{Li}\right)$. 其中, $E_{\mathrm{f}}$ 表示嵌入 $m$ 个 $\mathrm{Li}$ 后能形 成空位(包括 Mo 空位或 $\mathrm{O}$ 空位)所需要吸收的能量, 即 空位形成能. $E(\mathrm{~V})$ 表示嵌入 $m$ 个 $\mathrm{Li}$ 后形成空位的晶胞的 总能量. $u$ 表示空位原子的化学势; $E\left(\mathrm{MoO}_{2}+m * \mathrm{Li}\right)$ 表示 嵌入 $m$ 个 $\mathrm{Li}$ 的 $\mathrm{MoO}_{2}$ 晶胞的总能量. 图 5 给出了空位缺 陷计算模型示意图, 其中 $(\mathrm{a} \sim \mathrm{g})$ 分别反映为 $\mathrm{Mo}$ 空位和 $\mathrm{O}$ 空位形成能的计算过程.

计算结果表明, 在没有 $\mathrm{Li}$ 的情况下, Mo 和 $\mathrm{O}$ 的空 位形成能分别为 $3.22 \mathrm{eV}$ 和 $9.99 \mathrm{eV}$. 空位形成能较大, 不易形成空位，而当嵌入一个 $\mathrm{Li}$ 后, $\mathrm{Li}$ 周围的 Mo 的空 位形成能大大降低, 为 $0.96 \mathrm{eV}$, 而周围 $\mathrm{O}$ 的空位形成能 变化不明显, 为 $9.95 \mathrm{eV}$. 这进一步验证了 $\mathrm{Li}$ 的嵌入能 减弱 $\mathrm{Mo}$ 和 $\mathrm{O}$ 之间的相互作用这一观点.

基于以上研究结果, 本文进一步计算了 $\mathrm{Mo}$ 周围嵌 入多个 Li 情况下的 Mo 空位形成能, 计算结果如图 6 所 示, 当 Mo 周围嵌入 4 个 $\mathrm{Li}$ 时, 空位形成能为 $-0.75 \mathrm{eV}$, 即形成空位后的能量比未形成空位的能量低 $0.75 \mathrm{eV}$, 表明形成空位后更稳定. 这是由于 Mo 空位带一定量的 负电荷, 产生极化效应，使体系的能量大大升高，而嵌 入的 $\mathrm{Li}$ 由于电荷部分转移给 $\mathrm{O}$ 而带正电, 能够中和 $\mathrm{Mo}$ 空位的负电中心, 使体系的能量降低. 整体上来看, $\mathrm{Li}$ 嵌入的个数增加能减小空位形成能. 随着 Mo 空位周围 $\mathrm{Li}$ 增加到 4 个 Mo 空位形成能下降至最低, 其后, Mo 的 空位形成能又增加, 这与配位的理论是统一的.

综上所述, 一方面, 嵌入 $\mathrm{Li}$ 后的 Mo 空位形成能与 没有嵌入 $\mathrm{Li}$ 的 Mo 空位形成能相比大大降低, 这是由于 $\mathrm{Li}$ 的嵌入给 $\mathrm{O}$ 提供了电子, 使得 $\mathrm{Mo}$ 和 $\mathrm{O}$ 之间的共价作 


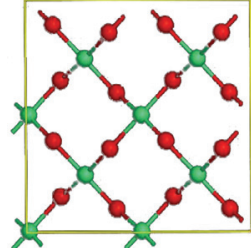

a

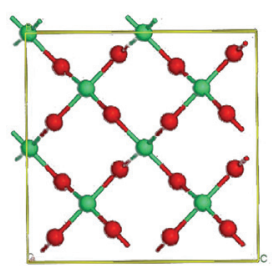

e

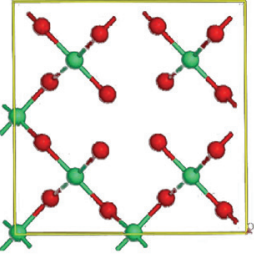

b

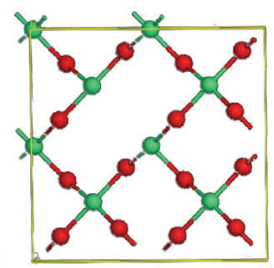

$\mathrm{f}$

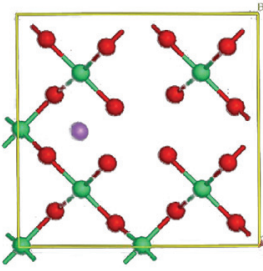

c

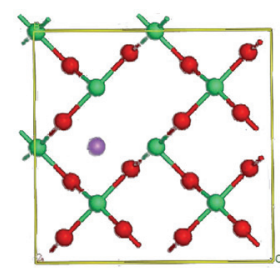

g
图 5 空位缺陷形成能计算模型示意图, 图(a), (e)表示理想 $\mathrm{MoO}_{2} ;(\mathrm{b})$, (f)分别表示无 Li 情况下的 Mo, O 空位; (c), (g)分别表示有一个 Li 的情 况下的 Mo, $\mathrm{O}$ 空位. 图中绿色为 $\mathrm{Mo}$ 原子, 红色为 $\mathrm{O}$ 原子, 紫色为 $\mathrm{Li}$ 原子

Figure 5 The model for calculating formation energy. The crystal of (a), (e) $\mathrm{MoO}_{2}$, (b), (f) $\mathrm{MoO}_{2}$ with Mo vacancy and $\mathrm{O}$ vacancy, (c), (g) $\mathrm{MoO}_{2}$ with Mo vacancy, $\mathrm{O}$ vacancy and $\mathrm{Li}$ interstitials. Green spheres are Mo atoms, red are $\mathrm{O}$ atoms and purple spheres represent $\mathrm{Li}$ atoms

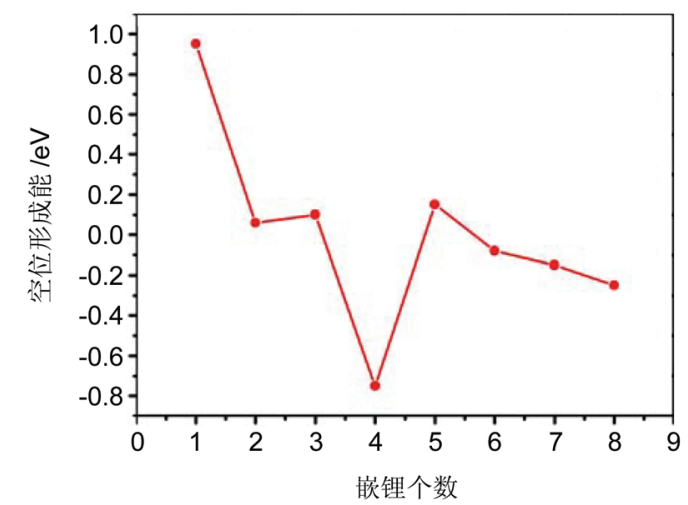

图 $6 \mathrm{Mo}$ 空位形成能与嵌入 $\mathrm{Li}$ 的个数的关系

Figure 6 The relationship of Mo vacancy formation energy with number of $\mathrm{Li}$ interstitials

用减弱, Mo 更容易形成空位. 另一方面嵌入的 Li 能减 弱 Mo 空位形成的极化, 使空位形成后的体系能量降低. 总之 $\mathrm{Li}$ 的嵌入能促进 Mo 空位的形成. 在嵌入多个 $\mathrm{Li}$ 的情况下, 嵌入 4 个 $\mathrm{Li}$ 时, Mo 空位形成能最低.

本文还计算了 Mo 空位的吸附能, 发现形成 Mo 空 位后, 空位中的单个 $\mathrm{Li}$ 的吸附能为 $3.26 \mathrm{eV}$, 可见 $\mathrm{Mo}$ 空位能为 $\mathrm{Li}$ 提供新的吸附位点. 随着循环次数增加, Mo 空位数量逐渐增加, $\mathrm{Li}$ 容量也就逐渐增加. 这很好地解 释了 $\mathrm{MoO}_{2}$ 电极材料随着循环次数的增加, 容量反而上 升的现象. 此外, 可以预见, 循环次数增加到一定数量 时, 由于 $\mathrm{MoO}_{2}$ 结构被较大程度的破坏, 将导致容量的 快速大幅衰减, 这与实验也是相符的.

\section{4 结论}

采用基于密度泛函的第一性原理计算，从微观的角 度利用不同的嵌锂结构考察了 $\mathrm{Li}$ 嵌入 $\mathrm{MoO}_{2}$ 电极材料中 的吸附情况和平均嵌入电压, 计算结果与实验结果符合 的很好. 本文还通过计算 $\mathrm{LiMoO}_{2}$ 结构的差分电荷密度、 电荷布居并结合 $\mathrm{Mo}$ 空位形成能综合地反映了 $\mathrm{MoO}_{2}$ 作 为电极材料循环容量反常特性的微观机理, 计算结果表 明 $\mathrm{Li}$ 的嵌入能减弱 $\mathrm{Mo}-\mathrm{O}$ 之间的相互作用，且能减小 Mo 空位带来的电荷极化效应, 促进 Mo 空位的形成. 进 一步, 形成的 Mo 空位能为 $\mathrm{Li}$ 的嵌入提供新的能量较低 的吸附位点, 从而提高了电极的储锂容量. 显见, Mo 空 位的形成对 $\mathrm{MoO}_{2}$ 电极材料晶体结构造成破坏, 储锂容 量会在一定循环次数后迅速消退, 与实验测量 30 次后容 量迅速下降的结果相符. 所以, 本文的计算结果对于阐 述 $\mathrm{MoO}_{2}$ 作为电极材料的特性, 尤其是其循环容量反常 特性是有意义的，同时也能指导电极材料的选择和改进.

\section{References}

[1] Arico, A. S.; Bruce, P.; Scrosati, B.; Tarascon, J. M.; Van Schalkwijk, W. Nat. Mater. 2005, 4, 366.

[2] Bruce, P. G.; Scrosati, B.; Tarascon, J.-M. Angew. Chem., Int. Ed. 2008, 47, 2930.

[3] Meduri, P.; Pendyala, C.; Kumar, V.; Sumanasekera, G. U.; Sunkara, M. K. Nano Lett. 2009, 9, 612.

[4] Hu, Y.-S.; Kienle, L.; Guo, Y.-G.; Maier, J. Adv. Mater. 2006, 18, 1421.

[5] Wu, C. Z.; Yin, P.; Zhu, X.; OuYang, C. Z.; Xie, Y. J. Phys. Chem. B 2006, 110, 17806.

[6] Li, H.; Fei, G. T.; Cui, P.; Jin, Y.; Feng, X. Y.; Chen, C. H. Chin. J. Chem. Phys. 2011, 24(3), 343. (李惠, 费广涛, 崔平, 金翼, 冯旭 勇, 陈春华, 化学物理学报, 2011, 24(3), 343.)

[7] Du, N.; Zhang, H.; Chen, B.; Wu, J. B.; Ma, X. Y.; Liu, Z. H.; Zhang, Y. Q.; Yang, D.; Huang, X. H.; Tu, J. P. Adv. Mater. 2007, 19, 4505 .

[8] Yao, W. L.; Yang, J.; Wang, J. L.; Nuli, Y. J. Electrochem. Soc. 2008, 155, A903.

[9] Varghese, B.; Reddy, M. V.; Zhu, Y. W.; Lit, C. S.; Hoong, T. C.; Rao, G. V. S.; Chowdari, B. V. R.; Wee, A. T. S.; Lim, C. T.; Sow, C. H. Chem. Mater. 2008, 20, 3360.

[10] Su, Z.; Xu, M.-W.; Ye, S.-H.; Wang, Y. L. Acta Phys.-Chim. Sin. 2009, 25(6), 1232. (粟智, 徐茂文, 叶世海, 王永龙, 物理化学学 报, 2009, 25(6), 1232.)

[11] Zhao, J. Z.; Tao, Z. L.; Liang, J.; Chen, J. Cryst. Growth Des. 2008, 8, 2799.

[12] Whittingham, M. S. Prog. Solid State Chem. 1978, 12, 41.

[13] Scrosati, B. J. Electrochem. Soc. 1992, 139, 2776.

[14] Lei, D. N.; Zhang, M.; Hao, Q. Y.; Chen, L. B.; Li, Q. H.; Zhang, E. D.; Wang, T. H. Mater. Lett. 2011, 65, 1154.

[15] Arrouvel, C.; Parker, S. C.; Islam, M. S. Chem. Mater. 2009, 21, 4778.

[16] Guo, B. K.; Fang, X. P.; Li, B.; Shi, Y. F.; Ouyang, C. Y.; Hu, Y. S.; Wang, Z. X.; Stucky, G. D.; Chen, L. Q. Chem. Mater. 2012, 24, 457.

[17] Hohenberg, P.; Kohn, W. Phys. Rev. 1964, 136, B864.

[18] Kohn, W.; Sham, L. J. Phys. Rev. 1965, 140, A1133.

[19] Kresse, G.; Hafner, J. J. Phys.: Condens. Matter 1994, 6, 8245.

[20] Kresse, G.; Joubert, D. Phys. Rev. B 1999, 59, 1758.

[21] Perdew, J. P.; Chevary, J. A.; Vosko, S. H.; Jackson, K. A.; Pederson, M. R.; Singh, D. J.; Fiolhais, C. Phys. Rev. B 1992, 46, 6671.

[22] Perdew, J. P.; Wang, Y. Phys. Rev. B 1992, 45, 13244.

[23] Brattas, L.; Kjekshus, A. Acta Chem. Scand. 1971, 25, 2783.

[24] Janotti, A.; Van de Walle, C. G. Phys. Rev. B 2007, 76, 165202.

(Cheng, B.; Fan, Y.) 